\title{
Myasthenia Gravis and Anesthesia Challenges
}

\author{
Harde Minal ${ }^{1}$, Bhadade Rakesh ${ }^{2}$
}

\begin{abstract}
Myasthenia gravis (MG) is an autoimmune disorder affecting nicotinic acetylcholine receptors at the post-synaptic site in the neuromuscular junction (NMJ). Myasthenia gravis presents with muscle weakness and fatigability of varying degrees affecting many muscle groups, mainly proximal skeletal muscles, ocular, respiratory, and bulbar. Myasthenia gravis has many implications for safe management of anesthesia. They are due to the disease process, association with other autoimmune diseases [diabetes mellitus (DM), thyroid disorders, systemic lupus erythematosis (SLE), rheumatoid arthritis (RA)], the medications used for treatment, potential for respiratory compromise, interaction of many anesthetic drugs in particular being resistance to suxamethonium, and high sensitivity to nondepolarizing muscle relaxants [neuromuscular blockers (NMB)]. Hence, detailed knowledge of the disease is necessary to the anesthesiologist for successful perioperative management and outcome. Keywords: Anesthesia, Muscle relaxants, Myasthenia gravis, Neuromuscular junction. Research and Innovation in Anesthesia (2019): 10.5005/jp-journals-10049-0070
\end{abstract}

\section{INTRODUCTION}

Myasthenia gravis (MG) is an autoimmune disease leading to varying degrees of proximal skeletal muscle weakness and fatigue affecting ocular, bulbar (muscles involved in speech, chewing, and swallowing), and respiratory muscles. It may be localized to specific muscle groups or generalized and weakness usually improves after rest. The reported incidence of MG worldwide is $0.3-2.8$ per $100,000{ }^{1}$ In MG, there is decrease in the numbers of acetylcholine receptors at the neuromuscular junction (NMJ) (postsynaptic site), which decreases the transmission of acetylcholine and nerve signal, hence deficient to trigger a muscle action potential. This results in weakness and repetitive stimulation results in fatigability (Fig. 1). Autoimmune conditions associated with MG are thyroid abnormalities, diabetes mellitus (DM), rheumatoid disease, systemic lupus erythematosis (SLE), pernicious anemia, ulcerative colitis, pemphigus, vitiligo, etc. ${ }^{2-4}$

\section{Clinical Classification of MG}

Osserman functionally and regionally graded the clinical severity of MG as follows (Table 1). ${ }^{4}$

\section{Clinical Features}

The principal presenting features of MG are weakness and easy fatigability of proximal skeletal muscles. The occurrence is related to age and sex, with female preponderance in second and third decades and male predominance in the sixth and seventh decades. ${ }^{3}$ Ptosis and diplopia are presenting features and in most cases may remain localized to the extraocular muscles. The symptoms are unique as with repetitive activity, weakness and fatigue usually increases and it improves after rest. In bulbar MG, facial muscles are affected, presenting with distinctive features like nasal tone to speech, flattening of smile crease, and weakness affecting the proximal limb. They also present with difficulty in chewing, swallowing, weak neck extensor muscles, and diaphragm. Undiagnosed MG may present with decreased exercise tolerance and overall tiredness that gets better after rest. ${ }^{4}$

Severe exacerbations seen in MG are myasthenic crisis and cholinergic crisis.

\begin{abstract}
'Department of Anesthesiology, Topiwala National Medical College and BYL Nair Charitable Hospital, Mumbai, Maharashtra, India

${ }^{2}$ Department of Medicine and Critical Care, Topiwala National Medical College and BYL Nair Charitable Hospital, Mumbai, Maharashtra, India

Corresponding Author: Harde Minal, Department of Anesthesiology, Topiwala National Medical College and BYL Nair Charitable Hospital, Mumbai, Maharashtra, India Phone: +91 9322294681, e-mail: minalharde@gmail.com
\end{abstract}

How to cite this article: Minal H, Rakesh B. Myasthenia Gravis and Anesthesia Challenges. Res Inno in Anesth 2019;4(2):36-39.

Source of support: Nil

Conflict of interest: None

Myasthenic crisis due to insufficient medication and cholinergic crisis due to excessive medication may lead to emergency severe episodes and both crises patient can present in a similar way. Patients have expressionless face, slack jaw, inability to support the head, nasal voice tone, and floppy body. Patients may present

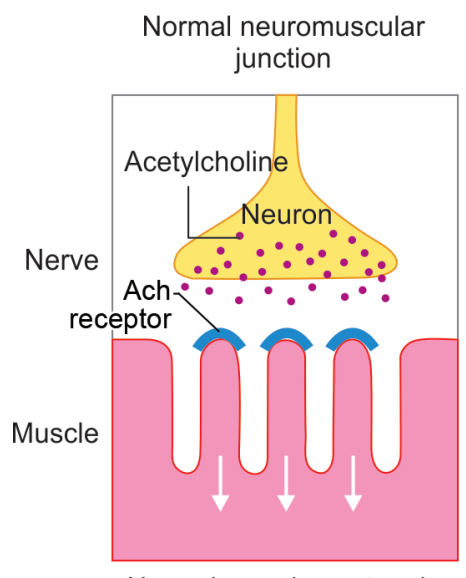

Normal muscle contraction
Neuromuscular junction in myasthenia gravis

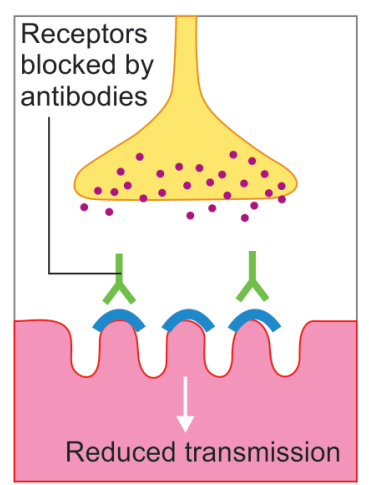

Impaired muscle contraction

Fig. 1: Comparison of normal neuromuscular junction and neuromuscular junction in myasthenia gravis

() The Author(s). 2019 Open Access This article is distributed under the terms of the Creative Commons Attribution 4.0 International License (https://creativecommons. org/licenses/by-nc/4.0/), which permits unrestricted use, distribution, and non-commercial reproduction in any medium, provided you give appropriate credit to the original author(s) and the source, provide a link to the Creative Commons license, and indicate if changes were made. The Creative Commons Public Domain Dedication waiver (http://creativecommons.org/publicdomain/zero/1.0/) applies to the data made available in this article, unless otherwise stated. 
Table 1: Clinical severity of myasthenia gravis (MG)

\begin{tabular}{ll}
\hline Grade & Severity \\
\hline Grade I & Only eyes affected \\
Grade IIa & $\begin{array}{l}\text { Mild generalized MG responsive } \\
\text { to treatment } \\
\text { Moderate generalized MG less } \\
\text { Grade IIb }\end{array}$ \\
Grade III & Severe generalized disease \\
Grade IV & Myasthenic crisis requiring \\
& mechanical ventilation \\
\hline
\end{tabular}

with bronchospasm, bronchorrhea, respiratory failure, diaphoresis, and cyanosis. An inability to cough along with absent gag reflex leads to an accumulation of secretions and patients are at risk for aspiration; also, inability to generate adequate ventilation may require mechanical ventilation. ${ }^{1-4}$

Cholinergic crisis in addition to above symptoms may present with miosis, excess salivation, lacrimation, urinary incontinence, diarrhea, Gl upset, and emesis called as SLUDGE syndrome. ${ }^{3,5}$

\section{DiAgnOSIS}

Anti-AchR antibodies are diagnostic for MG. A chest X-ray is needed to rule out aspiration pneumonia. A CT scan or MRI of the chest is indicated for diagnosing thymoma. Other investigations to exclude any associated autoimmune diseases.

In case of crises, an arterial blood gas ( $A B G)$ is necessary to diagnose respiratory failure (increased $\mathrm{PaCO}_{2}$ ) and ICU management.

The tensilon (edrophonium) challenge test is valuable in diagnosing MG and in differentiating among myasthenic and cholinergic crisis. Test dose of edrophonium is given only after the patient's airway is protected and ventilation is confirmed. Graded doses from 1 to $5 \mathrm{mg}$ may show obvious muscle strength improvement in 1 minute in myasthenic crisis. Watch for edrophonium-induced adverse effects (bradycardia, heart block, and asystole) and return of muscle weakness, respiratory distress, and pulmonary aspiration after the edrophonium effect is worn off. In cholinergic crisis patients, SLUDGE syndrome may be manifested and even maximum dose of edrophonium $(10 \mathrm{mg})$ fails to improve the muscle strength.

The ice pack test is done by keeping ice pack over an eyelid and will lead to improvement of the ptosis as cooling may improve neuromuscular transmission.

For confirmation of the diagnosis of MG, standard electromyography (EMG), single-fiber EMG, and acetylcholine receptor antibody assays can be done. The EMG testing shows decreased compound muscle action potential (CMAP) after a supramaximal single twitch and fade on tetanic stimulation $(>10 \%)$, which is analogous to a small dose of nondepolarizing relaxant. ${ }^{2-5}$

\section{MANAGEMENT}

Medical management of MG is mainly to improve neuromuscular transmission by drugs like anticholinesterases, immune suppression, and short-term immunotherapies. In selected cases, surgical thymectomy is needed. ${ }^{1,4}$

\section{Anticholinesterase Agents}

They are the first drug of choice for MG. Pyridostigmine (Mestinon $30 \mathrm{mg}$ orally) is the most extensively used anticholinesterase.
Beyond maximal dosing (120 mg), it may produce paradoxical weakness and after weeks of therapy strength improvement usually decreases.

Immunosuppressive drugs are used for continuing immunosuppression for long time in MG. They are prednisone, azathioprine, cyclosporine, mycophenolate mofetil, methotrexate, tacrolimus, and in refractory MG cyclophosphamide and rituximab.

Short-term immunotherapies include plasmapheresis (plasma exchange) and intravenous immune globulin (IVIg) and indicated in myasthenic crisis or for the preoperative optimization in patients undergoing thymectomy. Plasmapheresis helps by removing antibodies from the circulation. They produce rapid but short-term clinical improvement lasting for few days. ${ }^{1}$

Surgical thymectomy is indicated in patients who are poorly controlled by anticholinesterases. The purpose of thymectomy is to correct disturbance of immune regulation, reducing need of immunosuppressive agents, and induce remission. Thymectomy can be performed by open or thoracoscopic approach and is an elective procedure, which should be performed when the patient is stable. ${ }^{6}$

\section{Myasthenic Variants}

Transient neonatal myasthenia is observed in neonates born to myasthenic mothers (20\%). Neonates may have trouble in sucking and swallowing. They should be screened for ptosis and distressed breathing. It is usually self-limiting but if neonate is taken for any emergency surgery then anesthesia implications are same as in MG. Three main forms of MG are observed in children; they are juvenile, neonatal, and congenital. ${ }^{7}$

Lambert-Eaton myasthenic syndrome (LEMS) is a unusual disease affecting NMJ and is associated with malignancies, but it can be associated with autoimmune disorders. Patients with LEMS have muscle weakness caused by reduced acetylcholine release from presynaptic nerve terminals. Unlike MG, LEMS is very sensitive to both nondepolarizing and depolarizing NMBs and autonomic dysfunction is common. ${ }^{7}$

\section{Anesthetic Management}

Preoperative evaluation of the MG patient should be done in detail including age, sex, onset, duration, progress and severity of the disease, the presence of thymoma, the treatment regimen, and emergency exacerbations. Other disorders that may have association with MG, that is, thyroid abnormalities, DM, SLE, RA, and other autoimmune diseases, should be screened and optimum control is necessary.

For detailed assessment and optimization, patients should be admitted 2 days before the planned procedure. Evaluation of the severity of myasthenia must be done in detail. Bulbar or respiratory involvement compromises the patient's capability to shield the upper airway and cough and clear secretions postoperatively. ${ }^{4-6}$ Mediastinal compression syndrome (stridor, positional dyspnea, facial engorgement) due to thymoma should be screened. ${ }^{6}$ Careful medication history is crucial as many medications may provoke exacerbations (Table 2).

On detailed physical examination, voluntary and respiratory muscle strength should be precisely assessed. Baseline muscle strength should be assessed by objective methods like vital capacity, timed forward-arm abduction, and dynamometry and should be documented. Even though muscle weakness is predominant in MG patients, coordination and deep tendon reflexes are conserved. A thorough respiratory evaluation is must to rule out evidence 
Table 2: Medications to be used with extreme caution in myasthenia gravis

\begin{tabular}{ll}
\hline Antibiotics & $\begin{array}{l}\text { Fluoroquinolones, macrolides, } \\
\text { tetracycline, chloroquine, } \\
\text { aminoglycosides }\end{array}$ \\
Anti-arrhythmics miscellaneous & $\begin{array}{l}\text { Calcium channel blockers, } \\
\text { lidocaine, beta-blockers, } \\
\text { quinidine, muscle } \\
\text { relaxants, trimethaphan, } \\
\text { diphenylhydantoin, } \\
\text { procainamide } \\
\text { Lithium, adrenocorticotropic } \\
\text { hormone, chlorpromazine, } \\
\text { levothyroxine }\end{array}$ \\
\hline
\end{tabular}

Table 3: Predictive preoperative risk factors for prolonged postoperative ventilation

Disease duration $>6$ years
Concomitant pulmonary disease
Major surgery
Grades III and IV MG
Vital capacity $<40 \mathrm{~mL} / \mathrm{kg}$
Peak inspiratory pressure $<-25 \mathrm{~cm} \mathrm{H}_{2} \mathrm{O}$
Pyridostigmine dose $>750 \mathrm{mg} /$ day

of pneumonia due to inability to cough and retained secretions. They are of utmost concern with respect to postoperative outcome. For objective monitoring of respiratory reserve, measurement of forced vital capacity (FVC) at regular intervals is essential. ${ }^{6,8}$ Table 3 mentions preoperative factors associated with need for prolonged postoperative mechanical ventilation and morbidity. ${ }^{4-8}$

Preoperative investigations appropriate to surgical procedure should be done as patients with MG may be presented for any other elective or emergency surgery or thymectomy. Regular blood investigations, imaging, and pulmonary function tests are must. In case of a mediastinal thymoma, the extent of the respiratory obstruction (fixed or dynamic) to be evaluated by flow-volume loops, CT scan of the chest, and echocardiography. ${ }^{6}$ Documenting quantified respiratory muscle strength using forced vital capacity and negative inspiratory pressure is essential. These pulmonary function tests are crucial to decide postoperative airway management, mechanical ventilation, and extubation. ${ }^{6,8}$

Preoperative optimization is of utmost importance to distinctly reduce the risk of procedure, anesthesia, and for better patient outcome.

- Optimization of the patient's strength and especially respiratory function is most vital.

- Tapering of immunosuppressive agents to reduce possibility of infection.

- Anticholinesterase agents can be discontinued, or if continued adequate atropinization must be ensured.

- Plasmapheresis can be used for the preoperative optimization in patients undergoing thymectomy for short-term perioperative clinical improvement.

- Counseling and psychological support to the patient.

\section{Anesthesia Plan}

In MG patients, the anesthesia management plan should be tailor-made depending on procedure, emergency or elective, major surgery, and the severity of the MG. Regional anesthesia is considered safe when applicable and with reduced doses of the ester local anesthetic. General anesthesia is safe in MG patients if preoperative optimization is adequate. Keeping in mind resistance to suxamethonium and the sensitivity to NMBs, monitoring of neuromuscular transmission is vital. ${ }^{4-8}$

Anticholinergic premedication is must; however, anxiolytic, sedative, and opioid premedication in titrated doses should be used with vigilance, however, in patients with respiratory distress and bulbar involvement and mediastinal compression should be avoided. In patients who are on corticosteroid therapy for long duration, hydrocortisone $100 \mathrm{mg}$ iv at induction followed by 200 $\mathrm{mg} /$ day infusion should be administered. ${ }^{9}$

In patients with mediastinal thymoma, risks of potential airway obstruction, hypoxia, and cardiovascular collapse are known. Therefore, thorough preparedness for an awake intubation in the sitting position, maintaining spontaneous ventilation, is critical. Also keeping a rigid bronchoscope and a cardiopulmonary bypass standby is must for optimum airway and cardiovascular management. ${ }^{6}$

A standard balanced general anesthesia technique including intravenous or inhalational anesthetic agents without use of neuromuscular blocking agents (NMBAs) whenever possible is recommended. Total intravenous anesthesia (TIVA) with remifentanil for MG has been reported by some authors. ${ }^{10}$ As there is resistance to suxamethonium and the excessive sensitivity to NMBs, absolute omission of muscle relaxants can be done. As inhaled anesthetics may cause profound muscle relaxation in MG patients, maintaining deep level for tracheal intubation and surgery is possible with inhalational anesthesia. Also, desflurane and sevoflurane having low blood solubility, rapid elimination, and quick neuromuscular transmission recovery are agents of choice. Anticholinesterase medication affects the degree of relaxation and duration of action of NMBAs. If NMBAs are essential, the degree blockade should be monitored using a quantitative train-of-four (TOF) neuromuscular monitor (NMM). Atracurium doses can be predictably less in patients with MG if in the preinduction period there is presence of fade (T4/ $\mathrm{T} 1<0.9)$. If absolutely necessary, titrated small doses $(10-25 \%$ of the ED95) of preferably atracurium or other intermediate-acting muscle relaxants can be used with a NMM with TOF for intubation, surgical relaxation, and reversal. ${ }^{4-8}$ It is preferable to use the multimodal analgesia (MMA) technique using short-acting agents along with epidural or regional nerve blocks as they suppress sympathetic response to pain and maintain stable hemodynamic parameters. Regional analgesia also provides superior postoperative analgesia without sedation while maintaining pulmonary function. ${ }^{10,11}$

At the end of surgery whether to reverse residual neuromuscular blockade is controversial, but, if needed, reversal with sugammadex rather than neostigmine is recommended. As with neostigmine, cholinergic crisis in the postoperative period may be difficult to diagnose. Also, extubation after spontaneous recovery from NMBs by adequate clinical (sustained head-lift) and NMM parameters (TOF ratio of $>0.9$ ) is advocated. In MG patients with involvement of bulbar or respiratory muscle, the different NM response of peripheral vs bulbar muscles is more obvious. ${ }^{4-10}$ Therefore, it is indispensable that sustained muscle strength in respiratory 
muscle group in a wide awake patient must be confirmed prior to spontaneous ventilation and extubation.

\section{Postoperative Management}

In the postoperative period, ventilatory parameters need vigilant monitoring as these patients have propensity to develop respiratory failure. Long-duration mechanical ventilation may be required after thymectomy. ${ }^{6}$ Also unique problem encountered in MG patients is weakness after surgery and it may be due to crisis (myasthenic or cholinergic), nonanesthetic drugs that may interfere with neuromuscular transmission, and rarely due to residual effects of anesthetic drugs. ${ }^{1}$

For the safe administration of anesthesia in MG patients, comprehensive understanding of this disorder is vital. Optimal management and successful perioperative outcome in MG patients is possible with detailed knowledge and meticulous planning among the coordinated multispecialty team.

\section{References}

1. Sanders DB, Wolfe Gl, Benatar M, et al. International consensus guidance for management of myasthenia gravis: executive summary. Neurology 2016;87(4):419-425. DOI: 10.1212/ WNL.0000000000002790.

2. Murai $\mathrm{H}$. Japanese clinical guidelines for myasthenia gravis: putting into practice. Clin Exp Neuroimmunol 2015;6(1):21-31. DOI: 10.1111/ cen3.12180.
3. Conti-Fine BM, Milani M, Kaminski HJ. Myasthenia gravis: past, present, and future. J Clin Invest 2006;116(11):2843-2854. DOI: 10.1172/ $\mathrm{JCl} 29894$.

4. Thanvi BR, Lo TCN. Update on myasthenia gravis. Postgrad Med J 2004;80(950):690-700. DOI: 10.1136/pgmj.2004.018903.

5. Postevka E. Anesthetic implications of myasthenia gravis: a case report. AANA J 2013;81(5):386-388. DOI: 10.23937/23774630/2/1/1022.

6. Başkan S, Örnek D, Güney A, et al. Management of anaesthesia in a patient with myasthenia gravis. Int J Anesthetic Anesthesiol 2015;2:022.

7. El-Dawlatly AA. Anaesthesia for thoracoscopic thymectomy: modified non-muscle relaxant technique - case reports. Middle East J Anaesthesio 2007;19(1):219-224.

8. Katz JA, Murphy GS. Anesthetic consideration for neuromuscular diseases. Curr Opin Anaesthesiol 2017;30(3):435. DOI: 10.1097/ ACO.0000000000000466.

9. Gritti P, Sgarzi M, Carrara B, et al. A standardized protocol for the perioperative management of myasthenia gravis patients. Experience with 110 patients. Acta Anaesthesiol Scand 2012;56(1):66. DOI: 10.1111/j.1399-6576.2011.02564.x.

10. Marik PE, Varon J. Requirement of perioperative stress doses of corticosteroids: a systematic review of the literature. Archives of Surgery 2008;143(12):1222-1226. DOI: 10.1001/archsurg.143.12.1222.

11. Bergmann I, Szabanowski T, Bräuer A, et al. Remifentanil added to sufentanil-sevoflurane anaesthesia suppresses hemodynamic and metabolic stress responses to intense surgical stimuli more effectively than high-dose sufentanil-sevoflurane alone. BMC Anesthesiol 2015;15(1):3. DOI: 10.1186/1471-2253-15-3. 